\title{
La grève des ouvriers de la construction navale à Québec (1840)
}

\section{Robert Tremblay}

Volume 37, numéro 2, septembre 1983

Travailleurs et mouvements sociaux

URI : https://id.erudit.org/iderudit/304155ar

DOI : https://doi.org/10.7202/304155ar

Aller au sommaire du numéro

Éditeur(s)

Institut d'histoire de l'Amérique française

ISSN

0035-2357 (imprimé)

1492-1383 (numérique)

Découvrir la revue

Citer cet article

Tremblay, R. (1983). La grève des ouvriers de la construction navale à Québec (1840). Revue d'histoire de l'Amérique française, 37(2), 227-239.

https://doi.org/10.7202/304155ar d'utilisation que vous pouvez consulter en ligne.

https://apropos.erudit.org/fr/usagers/politique-dutilisation/ 


\title{
LA GRÈVE DES OUVRIERS DE LA CONSTRUCTION NAVALE À QUÉBEC (1840)
}

\author{
ROBERT TREMBLAY \\ Dictionnaire biographique du Canada \\ Québec
}

Pour le prolétaire, la vie se résout en ces

trois mots: naître, souffrir et mourir.

La misère préside à la naissance, et

compagne inséparable, ne l'abandonne qu'à

sa mort, sauf de bien rares exceptions.

L'Écho de la fabrique (Lyon, France) 17 février 1833.

Cette phrase lancée par un ouvrier de la soie dans la foulée de la célèbre grève des Canuts lyonnais en 1833 n'est pas sans nous rappeler la condition des travailleurs de la construction navale de Québec qui, à la même époque, interrompent le travail pendant 18 jours afin de briser la corrélation fatale qui s'était établie entre l'accumulation capitaliste et l'accumulation de la misère.

Certains se demanderont pourquoi avoir choisi comme objet d'étude la grève de 1840 des charpentiers de navires à Québec. D'abord, il faut dire que la grève est fondamentalement un événement qui parle et dont on parle. Elle suscite le discours non seulement des gardiens de l'ordre mais aussi celui des journalistes, des témoins et des acteurs. La grève force l'attention, entretient l'inquiétude et oblige à l'enquête. Elle fait donc foisonner la matière documentaire. Par ailleurs, la grève est aussi un «fait social» qui multiplie les rapports entre les classes habituellement installées dans des compartiments séparés; chaque conflit de travail agit donc comme révélateur des rapports sociaux de production à

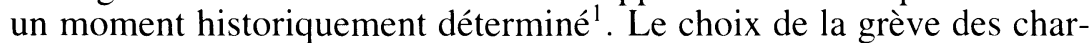
pentiers de navires n'est certes pas gratuit lui non plus. En effet, l'analyse de ce conflit vise à remettre en cause cette vision de l'historiographie québécoise qui prétend qu'après l'échec de l'insurrection patriote de 1837-1838, l'abattement du mouvement populaire fut tel qu'il ne se passa rien. Pourtant, l'examen de moments resserrés de l'histoire de cette période nous démontre la vivacité du jeune mouvement ouvrier: signalons la grève des terrassiers du canal Chambly $(1839)^{2}$, la grève

\footnotetext{
1 Pour approfondir la signification sociale de la grève ouvrière, deux excellents ouvrages sont à consulter: Michelle Perrot, Les ouvriers en grève, France, 1871-1890 (2 vol., Paris, Mouton, 1974) et Jocelyn Chamard, «Discours d'émancipation et discours de pouvoir: étude de la pensée des maîtres-tisserands de Lyon» (thèse de M.A., Département d'histoire, UQAM, 1980).

2 Canada, province du, Assemblée législative, Journal, 1843, appendice au troisième volume.
} 
de 1200 ouvriers de la construction navale à Québec et création d'un syndicat ouvrier (1840), la coalition des peintres de bâtiments à Québec $(1842)^{3}$ ainsi que la grève des 4000 terrassiers du canal Lachine et du canal Beauharnois $(1843)^{4}$. Il ne serait pas étonnant que ces luttes cruciales survenues au tournant des années 1840 se situent à l'amont d'un processus qui, selon la formule de Marx, fait basculer la communauté ouvrière de l'étape de «classe en soi», marquée par la formation matérielle de la classe ouvrière, à l'étape de «classe pour soi», caractérisée par l'émergence de l'élément immatériel que constitue la conscience ouvrières.

\section{L'industrie de la construction navale durant la première moitié du XIX ${ }^{e}$ siècle}

Durant la première moitié du XIXe siècle, la ville de Québec était un centre important de construction navale en Amérique du Nord britannique. De 1760 à 1825, les chantiers de Québec fournissaient à eux seuls $52 \%$ du tonnage global des vaisseaux construits dans le BasCanada $^{6}$. Comme sous le Régime français, cette industrie se pratiquait en bordure de la rivière Saint-Charles et dans la basse-ville de Québec. Parmi les firmes d'envergure, on retrouvait celle de John Goudie dans le faubourg Saint-Roch et celle de John Munn au pied du cap Diamant; dans les deux cas, on était parvenu à intégrer une série d'opérations auparavant dispersées. Ainsi, en 1831, le chantier Munn réunissait, dans une même unité de lieu, un moulin à scie, une forge, des ateliers où on travaillait le bois, une immense cour de construction, un entrepôt, une étable et un quai donnant sur le fleuve ${ }^{7}$.

En gros, l'industrie de la construction navale était organisée selon deux modes: celui de la sous-traitance et celui de la «manufacture» proprement dite. Dans le premier, le maître constructeur faisait figure d'auxiliaire du marchand, lui étant soumis par voie de contrat et lui étant redevable des installations et des matériaux nécessaires à la mise en chantier d'un navire. Dans le second mode, le maître constructeur assumait à lui seul la direction du procès de travail et le financement des opérations de production. Pour des raisons que l'on ignore, la forme «manufacture» deviendra prédominante à compter des années 1820 dans l'industrie de la construction navale à Québec. Affranchis du joug des marchands, les maîtres constructeurs n'étaient pas pour autant au bout

\footnotetext{
3 Eugène Forsey, Trades Unions in Canada, 1812-1902 (Toronto, UTP, 1982), 15.

4 H.C. Pentland, "The Lachine Strike of 1843», Canadian Historical Review (1948): 255 277; Raymond Boily, Les Irlandais et le canal de Lachine: La grève de 1843 (Montréal, Leméac, 1980).

5 K. Marx, Misère de la philosophie (Paris, UGE, 1965), 490. 1981): 244ss.

7 D.T. Ruddell, «Quebec City, 1765-1831: The Evolution of a Colonial Town» (thèse de Ph.D., Université Laval, 1981), 278.
} 
de leurs peines. Ainsi, durant les années 1830-1840, le resserrement du marché britannique de même que les mauvaises cotes attribuées par les compagnies d'assurance ${ }^{8}$ avaient eu pour effet d'abaisser le prix des navires canadiens. Néanmoins, en comprimant les dépenses au chapitre des frais d'entretien de la force de travail, les propriétaires de chantier parvenaient à retirer des profits bruts de l'ordre de $13 \%{ }^{9}$, dont une partie allait en commission à des agents financiers de Londres ${ }^{10}$. En 1834, pour les 16 vaisseaux construits à Québec d'une valeur de $£ 84037$, seulement 229446 étaient consacrées aux coûts en main-d'oeuvre tandis que $£ 43615$ servaient à l'achat de produits importés d'Angleterre (cuivre, fer, cordage, voiles, etc.) et à l'approvisionnement en bois de la colonie ${ }^{11}$. L'industrie de la construction navale se heurtait donc à la fragilité de son système d'accumulation qui reposait essentiellement sur une pression à la baisse de la masse salariale.

Un système tout aussi structuré régissait l'exécution du travail dans les chantiers. Ainsi, avec les années, il s'était développé un procès de travail pouvant concilier la complexité des tâches reliées à la construction navale et le caractère collectif de cette mise en oeuvre ${ }^{12}$; l'équipe de chantier constituait le pivot de ce mécanisme de production ${ }^{13}$. En général, les équipes de travail revêtaient une forme pyramidale ou hiérarchique au sommet de laquelle on retrouvait le contremaître, chargé de la supervision et de l'embauche de la main-d'oeuvre, suivi d'un contingent d'ouvriers qualifiés (charpentiers, menuisiers, calfats, forgerons, peintres, etc.) assignés au travail de montage, de pontage et de finition des navires puis, au bas de l'échelle, un groupe d'ouvriers sans qualification (apprentis et journaliers), réduits au rôle de simples auxiliaires de leur maître respectif. Il va sans dire qu'au cours des années 1820-1840, la masse des ouvriers non qualifiés se gonflera au détriment des charpentiers de navires qui verront leur emploi de plus en plus

\footnotetext{
${ }^{8}$ Adam Shortt et A.G. Doughty, Canada and its provinces (23 vol., Toronto, 1913-1917), $\mathrm{X}: 577$.

9 Cette estimation a été réalisée à partir de données, extraites de La Gazette de Québec du 12 février 1834, sur les coûts et la valeur de la production dans la construction navale.

10 Les commissions versées aux agents représentaient environ $5 \%$ du prix de vente d'un navire; on faisait habituellement appel aux agents pour trouver des débouchés à la production des chantiers navals ou tout simplement pour obtenir un emprunt sur le marché financier de Londres.

${ }^{11}$ La Gazette de Québec, 12 février 1834, 18 janvier 1837. On rapporte qu'il en coûtait $35 \%$ plus en matériaux pour construire un navire à Québec que dans les fameux chantiers de la rivière Clyde, en Écosse; le prix élevé du bois de construction dans la colonie et les frais de douane sur les produits importés d'Angleterre expliquent cette situation. Voir à cet effet J.S. MacMillan et A.H.J. Richardson, «James Dunlop», Dictionnaire biographique du Canada, vol. V (à paraître) et Bas-Canada, Chambre d'Assemblée, Journaux, 28 janvier, 9 mars 1825.

12 Il nous a été impossible de mesurer jusqu'à quel point les procédés de travail se sont transformés sous l'impact de l'introduction de machines à vapeur ou de treuils mécaniques par des propriétaires de chantiers, tels John Goudie et John Bell, entre 1810 et 1820.

13 Pour plus de détails au sujet des équipes de chantier, on peut consulter: D.T. Ruddell, «Quebec City, 1765-1831...», 296-297.
} 
menacé par cette main-d'oeuvre à bon marché ${ }^{14}$. Comme l'a fait remarqué l'historien Richard Rice, le chantier naval était, durant la première moitié du $\mathrm{XIX}^{\mathrm{e}}$ siècle, à l'avant-garde des entreprises coloniales de production non seulement en terme de capitaux et de moyens de production mis en oeuvre, mais aussi en terme de division technique du travail et de discipline.

\section{La condition ouvrière}

Les monographies du XIX $\mathrm{X}^{\mathrm{e}}$ siècle présentent souvent les travailleurs de la construction navale comme des gens de plein air échappant à l'uniformité monotone des ateliers ${ }^{15}$. Il est vrai que le cycle saisonnier de l'activité productive dans cette industrie leur donne parfois des allures de nomades et que la possession des outils leur confère une certaine indépendance; mais faut-il pour autant conclure que ces ouvriers en soient arrivés à se soustraire, même partiellement, à la condition de prolétaires? Pas du tout. La précarité de la vie quotidienne, le désarroi devant les accidents ou autres difficultés de l'existence ainsi que la hantise du chômage constituaient le lot de ces ouvriers. N'oublions pas que les 2860 ouvriers ${ }^{16}$ travaillant dans les 11 chantiers navals de Québec au cours de l'hiver 1840-1841 concouraient au support social d'environ 10000 personnes, soit un peu plus de $20 \%$ de la population de la ville de Québec à l'époque. On comprend donc que les maigres salaires consentis à la fin de l'année $1840^{17}$, de même que l'escalade du prix des denrées étaient lourdement ressentis et contribuaient à raviver ce vieux sentiment de «l'obsession alimentaire» parmi la communauté ouvrière reliée à l'industrie de la construction navale. D'ailleurs, un cri d'alarme avait déjà été lancé un an plus tôt par les membres du syndicat des typographes de Québec dans une pétition qui déclarait en substance:

La cherté du coût de la vie à Québec a contraint depuis longtemps nos femmes à grapiller sou par sou, à économiser sur tout. Leurs

14 En général, on recrutait cette catégorie de travailleurs parmi la communauté paysanne des régions avoisinantes de Québec, bien qu'occasionnellement on eût recours aux immigrants venant tout juste de débarquer. Ainsi, on rapporte qu'en 1821 et 1822 , plus de 700 immigrants irlandais auraient travaillé temporairement à la construction navale dans les chantiers de Québec. Voir à ce sujet: Fernand Ouellet, Le Bas-Canada, 1791-1840; changements structuraux et crise (Ottawa, 1976), 220.

15 Cette vision idyllique de l'ouvrier du bâtiment se retrouve également en France pendant tout le $\mathrm{XIX}^{\mathrm{e}}$ siècle. L'ouvrage de Michelle Perrot, Les ouvriers en grève, France, 1871-1890, 377-379, nous renseigne de façon éloquente sur ce phénomène.

16 Quebec Mercury, 10 avril 1841; le même journal, en date du 11 février 1840, nous fournit une estimation de la population ouvrière sur les chantiers de Québec, de 1818 à 1840.

17 Il est à noter que le salaire moyen d'un charpentier de navire et d'un calfat était passé de 7 shillings/jour à l'été de 1839 à 3 shillings/jour à la fin de l'année 1840. Voir à cet effet: Narcisse Rosa, La construction de navires à Québec et ses environs (Québec, 1897), 10. D'ailleurs, tout au long du XIX ${ }^{\mathrm{e}}$ siècle, il existe en Amérique du Nord Britannique une tradition qui consiste à abaisser les salaires du quart ou même de la moitié en hiver. Dans un article intitulé «The Seasonal Contours of the Pre-Industrial Poverty in BNA, 1815-1860», CHA, Historical Papers (1974), l'historienne Judith Fingard constate que cette coutume persistera au point que la Commission d'enquête sur les relations entre le capital et le travail en fera mention en 1889 comme une des principales sources d'appauvrissement saisonnier chez les travailleurs. 
efforts n'empêcheront pas la grande majorité d'entre nous d'être criblés de dettes cet hiver... ${ }^{18}$

On estime qu'à la veille de la grève de 1840 , un charpentier de navires devait consacrer plus de la moitié de son salaire de 3 shillings/jour pour combler les besoins élémentaires de sa famille en nourriture ${ }^{19}$. De même, le bois de chauffage, qui se détaillait 12 shillings par corde, pouvait grignoter jusqu'à $20 \%$ des gages de cette catégorie de travailleurs pendant les mois d'hiver. Ainsi, apparaît un des traits saillants du budget ouvrier: sa vulnérabilité devant les périodes d'intermittence du travail. En raison des caprices du climat, l'activité des chantiers navals était régulièrement ponctuée d'interruptions momentanées, acculant la communauté ouvrière à de nombreuses privations. Plus dramatiques s'avéraient les accidents de travail ou la mise en chômage prolongé, lesquels facteurs réduisaient des familles entières à la mendicité. Les accidents de travail survenaient surtout au début de l'hiver lorsqu'il fallait manipuler d'immenses pièces de bois dans des conditions météorologiques dangereuses ${ }^{20}$ ou lorsqu'il fallait rattraper les retards dans les délais de livraison d'un navire par une augmentation des heures et de la cadence de travail. Quant au chômage, il sévissait en période de stagnation de l'activité sur les chantiers, conséquence de la contraction du marché britannique comme ce fut le cas au cours de la décennie 1840-1850. Pour les ouvriers de la construction navale qui vécurent cette crise, le problème du licenciement se posait avec une grande acuité, puisqu'on ne pouvait compter obtenir un emploi temporaire comme débardeur au port de Québec depuis que les matelots étaient retenus à bord pour effectuer le travail de déchargement ${ }^{21}$. Refoulée et emprisonnée dans le faubourg Saint-Roch, cette population ouvrière vivait les pires conditions sociales (insalubrité, disettes, etc.) pouvant exister dans la ville

\footnotetext{
1976), 40 .

18 Charles Lipton, Histoire du syndicalisme au Canada et au Québec (Montréal, Parti pris,

19 Cette évaluation a été réalisée à l'aide de l'indice des prix de denrées de consommation courante contenu dans les Rapports annuels du Quebec Board of Trade, 1832-1842, déposés aux ANQ-Q. Cette même source nous apprend que les charpentiers de navires de Québec figuraient parmi les ouvriers les moins bien rémunérés en 1840. La plupart des ouvriers qualifiés (forgerons, maçons, charpentiers de bâtiments, etc.) de Québec gagnaient au-dessus de 5 shillings/jour à cette époque.

Le journal Le Canadien (Québec) du 4 décembre 1840 fait état d'un accident survenu sur le chantier de M. Oliver dans le faubourg Saint-Roch: «Thomas Rostevin, 18 ans, apprenticharpentier fut tué par une pièce de bois qui tomba sur lui d'une hauteur assez considérable. Cette pièce l'écrasa si horriblement que lorsqu'on parvint à lui, il avait déjà expiré».

${ }_{21}$ Fernand Ouellet, Histoire économique et sociale du Québec, 1760-1850 (2 vol., Montréal, Fides, 1971), II: 504. Cette situation dramatique de l'emploi favorisait la mise sur pied de nouveaux systèmes d'exploitation de la force de travail. Ainsi, en 1842, John Munn proposa d'ouvrir ses chantiers pour la construction de «barges» qui étaient requises par les entreprises de canalisation dans le Bas et le Haut-Canada; toutefois, les ouvriers intéressés devaient consentir à travailler bénévolement avec une promesse plus ou moins vague d'un partage éventuel des bénéfices. Voir à ce sujet le Quebec Mercury du 27 décembre 1842. À la fin des années 1840, de nombreux ouvriers de la construction navale oeuvreront aux travaux d'aqueduc ou comme journaliers dans les carrières à la suite de leur licenciement.
} 
de Québec ${ }^{22}$. En dépit des opérations de philanthropie bourgeoise visant essentiellement à sédentariser la classe ouvrière en fonction des besoins futurs du capital - , cette ségrégation de classe au niveau de l'espace urbain était propice à aiguiller le monde ouvrier vers une prise de conscience de ses intérêts collectifs.

\section{La mise en mouvement des ouvriers de la construction navale en décembre 1840 ou le scénario d'une grève}

Conséquence du lent développement de la production capitaliste dans le Bas-Canada avant 1840, la résistance ouvrière apparaît souvent sous une forme primitive ${ }^{23}$ et se caractérise par son éparpillement. De plus, la composition de la classe ouvrière changeait trop rapidement, sous l'effet des migrations internes provoquées par les emplois saisonniers, pour que soit organisé un véritable mouvement contre les inégalités sociales dans les villes. Mais avec le débrayage des ouvriers de la construction navale en 1840, débute une première grève de masse dont il convient de rappeler le scénario d'ensemble ${ }^{24}$. Ainsi, au début du mois de décembre 1840, les principaux constructeurs de navires de Québec, anticipant de nouvelles commandes pour l'hiver, avaient conclu une alliance en vue d'abaisser les salaires de 4 à 3 shillings par jour; dans un réflexe de défense, 1200 ouvriers - surtout des charpentiers, des bûcheurs et des journaliers - , provenant des divers chantiers de Québec, entamèrent immédiatement une grève pour protester contre ce qu'ils avaient surnommé si justement «le pacte de la famine» ${ }^{25}$. Dès le 3 décembre, tous les chantiers étaient fermés, et sous l'invitation des charpentiers de navires, les ouvriers en grève se réunirent dans une école du faubourg Saint-Roch afin de coordonner leur action et d'organiser un consensus autour de revendications communes. Il fut convenu que la cessation du travail allait se poursuivre (tout l'hiver au besoin) tant que les salaires ne seraient pas revenus à 4 shillings par jour. Malgré les interdictions de la loi, on résolut d'organiser une collecte (qui recueillit $£ 50$ en 24 heures) pour soutenir les grévistes étant le plus dans le besoin. Enfin on accepta la formation d'un comité de 21 personnes dont le mandat consistait à communiquer les doléances des ouvriers aux constructeurs de navires. Au fil des événements, les ouvriers en grève

22 Pour obtenir une description détaillée de ces conditions sociales, on peut consulter le journal L'Artisan (Québec), 12 décembre 1842 et le Quebec Mercury, 10, 27 décembre 1842.

23 Nous entendons par «formes primitives» de la résistance ouvrière, tous les actes individuels et spontanés (vol, absentéisme, insubordination, sabotage, etc.) qui témoignent d'une remise en question de l'autorité patronale; il faut voir dans ces initiatives isolées, la manifestation d'un instinct de combativité ouvrière typique des premières phases du processus d'industrialisation capitaliste.

24 Signalons qu'en 1741, une grève avait éclaté sur les chantiers royaux de Québec et que l'intendant Hocquart avait vite fait d'écraser cette «mutinerie» en emprisonnant les auteurs et en les remplaçant par des ouvriers venus de Rochefort, en France. Voir à ce sujet: S.B. Ryerson, The Founding of Canada (Toronto, 1960), 154.

${ }^{25}$ Le Canadien, (Québec), 4 décembre 1840; Le Fantasque (Québec), 10 décembre 1840. 
tentèrent d'élaborer un réseau d'alliance parmi la population de Québec, en cherchant l'appui des autres «corps de métier», des petits commerçants et des intellectuels comme le journaliste Napoléon Aubin ${ }^{26}$.

En dépit du caractère structuré de ce mouvement de masse, on ne put prévenir l'action spontanée et la violence, tant l'exaspération des ouvriers de la construction navale était grande. Ainsi, le 8 décembre, une émeute éclata près des chantiers de la firme Munn, et le lendemain, des actes de pillage et de vol furent commis sur les chantiers d'Edward Oliver dans le faubourg Saint-Roch ${ }^{27}$. Quoi qu'il en soit, le 12 décembre 1840 , après 18 jours de conflit, les grévistes parvinrent à briser le monopole exercé par les constructeurs de navires sur le prix du travail et obtinrent que leur salaire fût reporté à 4 shillings par jour. À l'occasion d'une manifestation tenue ce jour-là à Québec, plus de 800 ouvriers de la construction navale vinrent présenter une adresse à George Black pour avoir été le premier à ouvrir son chantier au prix réclamé ${ }^{28}$.

Même si un des traits dominants de cette grève fut son caractère défensif, elle aura permis aussi à une première génération de prolétaires de prendre conscience de leur pouvoir et de leur importance, comme l'atteste ce commentaire paru dans le journal Le Fantasque: «Les bienfaits apportés à la classe des charpentiers par cette ligue momentanée, devraient leur montrer la nécessité de former une association permanente.» ${ }^{29}$

\section{La naissance du syndicalisme chez les charpentiers de navires}

L'élément le plus significatif de l'éveil de la conscience ouvrière demeure sans aucun doute la naissance de la Société amicale et bienveillante des charpentiers de vaisseaux de Québec, dans le sillage de la grève de 1840. Malgré les apparences d'une société de secours mutuel, cet organisme était voué d'abord à une fonction syndicale d'encadrement de l'action ouvrière ${ }^{30}$; en témoignent, ces avis lancés à quelques charpentiers de navires et journaliers des chantiers de Saint-Roch afin qu'ils respectent l'ordre de grève sous peine «d'être expulsés de la Société ou d'être jugés indignes d'y être admis» ${ }^{31}$.

L'idée de se regrouper au sein d'une «union ouvrière» avait vu le jour peu après le déclenchement de la grève, lors d'une réunion des

\footnotetext{
26 Le Canadien (Québec), 4, 7 décembre 1840; Quebec Mercury, 10 décembre 1840; Le Fantasque (Québec), 10 décembre 1840.

27 ANQ-Q, Registres de la prison de Québec, dossiers criminels, 1840-1841.

28 Le Canadien (Québec), 21, 28 décembre 1840; Montreal Gazette, 26 décembre 1840.

29 S.B. Ryerson, Le Capitalisme et la Confédération (Montréal, Parti pris, 1972), 234.

30 En vertu du Common Law, l'existence de coalitions ouvrières était perçue comme un acte de conspiration; c'est pourquoi, les activités des premiers syndicats demeuraient semi-clandestines et se présentaient sous le couvert de sociétés de secours mutuel. D'ailleurs, les caisses de secours en cas de maladie ou d'accident camouflaient souvent une caisse de résistance pour les ouvriers en grève.

31 Le Canadien (Québec), 21 décembre 1840.
} 
charpentiers de navires tenue dans le faubourg Saint-Roch, le 12 décembre 1840. On invoquait alors la nécessité de «maintenir [ce corps de métier] dans le degré d'influence qu'il doit occuper dans la société» ${ }^{32}$. Par la même occasion, on avait élu un conseil de 12 membres - dont François Giffard assumait la présidence - chargé de préparer les règlements de la Société et de promouvoir les vues de la base. Un des premiers objectifs du comité avait consisté à établir un front unique d'ouvriers, assez puissant pour faire une opposition vive et constante aux coalitions patronales dans l'industrie de la construction navale; dans cette optique, on avait convenu de pratiquer une ouverture, dans le recrutement, à toutes les catégories de travailleurs de cette industrie, en autant qu'ils fussent résidents du Bas-Canada et âgés de 15 à 50 ans $^{33}$. Toutefois, en raison de la prépondérance des charpentiers de navires $75 \%$ des effectifs $-{ }^{34}$ au sein de cette organisation syndicale qui comptait 255 membres en janvier 1841, on retint l'idée qu'il fallait également lutter contre la concurrence des immigrants et de la maind'oeuvre non qualifiée sur les chantiers ${ }^{35}$. Il apparaît donc que les intentions se profilant derrière la mise sur pied de la Société des charpentiers de vaisseaux furent profondément marquées par cette double conscience, prolétarienne et corporatiste, qui se développait dans les centres où la production capitaliste n'avait pas encore totalement révolutionné les vieux procès de travail à base artisanale.

Une fois réunie sous la bannière syndicale, les ouvriers de la construction navale eurent recours à un spécialiste de la pratique du droit afin de les conseiller dans leurs récriminations. Le 18 décembre 1840 , ils désignèrent le notaire Joseph Laurin ${ }^{36}$ pour occuper le poste de secrétaire de la Société des charpentiers de vaisseaux et lui confièrent entre autre le mandat de rédiger l'acte assurant une couverture légale aux activités du syndicat. Celui-ci devait, en outre, tenir les minutes des réunions du conseil et informer l'assemblée sur les droits de ses membres, notamment en situation de grève. D'ailleurs, en acceptant sa charge, le notaire Laurin avait déclaré qu'il était prêt à lutter contre le monopole des maîtres constructeurs et que la «fermeté finirait par l'emporter sur l'avarice sordide de ces spéculateurs de Québec» ${ }^{37}$.

Au chapitre de la prise de décision, l'assemblée des membres de la Société des charpentiers de vaisseaux disposait de la pleine souve-

32 Ibid., 16 décembre 1840.

33 Voir l'acte qui créait officiellement la Société amicale et bienveillante des charpentiers de vaisseaux de Québec, conservé aux ANQ-Q, dans le minutier du notaire Joseph Laurin, en date du 5 janvier 1841 .

34 Ibid.

35 Forsey, Trade Union in Canada, 1812-1902, 15; Ouellet, Histoire économique et sociale du Québec, II: 501 .

36 Pour en savoir davantage sur ce précurseur des conseillers juridiques syndicaux, on doit consulter Lucie Bouffard et Robert Tremblay, «Joseph Laurin», Dictionnaire Biographique du Canada (Québec, 1982), vol. XI: 549 ss.

37 Le Canadien, 21 décembre 1840. 
raineté. Les résolutions étaient adoptées à la majorité des voix et, en cas d'égalité, le président de la Société pouvait utiliser son droit de suffrage prépondérant. Le côté avant-gardiste des règlements de ce syndicat tenait en grande partie à l'introduction du principe de la responsabilité des membres du conseil de direction à l'endroit de l'assemblée. Ainsi, cette dernière pouvait révoquer en tout temps un «officier» pour malversation ${ }^{38}$. Comme le soulignera Médéric Lanctôt quelques années plus tard, les premières organisations syndicales étonnent par leur structure éminemment démocratique pour l'époque ${ }^{39}$. La mise sur pied de la Société des charpentiers de vaisseaux nous révèle en définitive les aptitudes de la classe ouvrière à gérer ses propres intérêts ${ }^{40}$; mais audelà de cette constatation, il faut voir que les mécanismes de fonctionnement et les principes fondamentaux de la démocratie étaient introduits au sein de l'organisation des charpentiers de navires par un sujet extérieur, le notaire Laurin, à qui on avait délégué la responsabilité de rédiger l'acte d'incorporation de la Société comprenant les statuts et les règlements. D'ailleurs, de récentes études sur la classe ouvrière en France, de 1830 à 1848 , ont démontré que l'idéologie populaire de cette époque est composée d'éléments inhérents comme le sentiment d'être exploité et d'éléments dérivés (ou extérieurs) comme la religion ou l'idée de la souveraineté populaire.

\section{L'État-gendarme et la classe ouvrière}

Abstraction faite des revendications strictement économiques en faveur d'un salaire équitable, la grève des ouvriers de la construction navale de Québec constituait également un acte de résistance, voire même un défi, au droit bourgeois. En refusant solidairement de vendre leur force de travail en deçà de conditions acceptables, ces ouvriers en grève remettaient en question la notion bourgeoise du «libre contrat individuel» - qui occulte l'inégalité dans la relation patron-employé - et proclamaient implicitement qu'un contrat n'a de valeur légale qu'avec la collectivité des travailleurs ${ }^{41}$. La mise sur pied de la Société des charpentiers de vaisseaux exprimait aussi, à sa manière, un refus de la légalité bourgeoise, dans la mesure où l'on passait outre à un interdit touchant le droit d'association. En effet, selon les combination acts, votés au Parlement de Londres en 1800 et mis en vigueur dans la colonie peu de temps après, tout individu se concertant avec d'autres

\footnotetext{
38 ANQ-Q, Minutiers, Joseph Laurin, 5 janvier 1841 385.

Margaret Heap, «La grève des charretiers à Montréal», RHAF, 31, 3 (décembre 1977):

40 Signalons que la Société des charpentiers de vaisseaux survivra à la crise des années 1840 dans l'industrie de la construction navale; en 1850, elle sera admise à l'incorporation (Voir: Canada, province du, Assemblée législative, Statuts, 1850, c. 127) et, en 1867, elle sera à l'origine d'une autre grève dans ce secteur d'activité (Voir: Jean Hamelin et al., Répertoire des grèves dans la province de Québec au XIX ${ }^{e}$ siècle (Montréal, 1970), 19 ss.).

${ }_{41}$ La question de l'existence d'une «légalité ouvrière» est abordée exhaustivement dans la thèse de J. Chamard, «Discours d'émancipation et discours de pouvoir...», 113-116.
} 
dans le but de revendiquer de meilleurs gages ou une diminution des heures de travail pouvait être inculpé pour conspiration criminelle. De même, les souscriptions en argent et les assemblées tenues à ces fins étaient considérées comme autant de délits au terme de la loi ${ }^{42}$. Comme beaucoup d'autres grèves du XIX ${ }^{\mathrm{e}}$ siècle, l'affrontement de décembre 1840, survenu dans l'industrie de la construction navale, révèle en latence deux conceptions antagoniques du droit et de la liberté; c'est d'ailleurs dans ce contexte que l'État sera appelé à jouer un rôle décisif.

Pressé par cette turbulence sociale à Québec, l'État dut intervenir au moyen d'un dispositif de répression juridico-politique s'orientant sélectivement contre la classe ouvrière. Ainsi, à la suite des émeutes du 8 et du 9 décembre sur les chantiers navals, cinq des «soi-disant meneurs ${ }^{43}$ furent appréhendés et condamnés par les juges de la Cour des sessions de la paix du district de Québec à des peines variant de 10 jours d'emprisonnement à 4 mois de travaux forcés ${ }^{44}$. Ces derniers s'empressèrent également de nommer John Munn, propriétaire de chantier, à la tête du comité de la police du guet, qui comprenait alors 140 hommes à Québec ${ }^{45}$. Probablement en raison du lobbying exercé par les maîtres constructeurs de navires et les marchands de Québec, l'Assemblée législative, nouvellement rétablie, adopta en 1841 la «loi relative aux dommages causés malicieusement à la propriété» ${ }^{46}$. Cette mesure visait à punir, par des sentences d'emprisonnement à vie ou d'exil, les actes de sabotage commis notamment contre des vaisseaux en construction. C'est cependant à l'article 25 que la loi prenait tout son sens, puisqu'on sanctionnait le droit d'établir une preuve de culpabilité sur le principe de l'intention malicieuse; dans cette optique, toute action de masse et particulièrement la résistance par la grève, pouvait être assimilée à l'intention d'endommager la propriété du capital ${ }^{47}$. Il va sans dire que, déjà à cette époque, le pouvoir d'État constituait un des remparts de la bourgeoisie lorsqu'elle se sentait moindrement lésée par l'agitation ouvrière.

\section{Discours de pouvoir et réplique ouvrière}

À bien des égards, la grève au XIX ${ }^{\mathrm{e}}$ siècle est le lieu privilégié où se déploie un rapport de force non seulement dans la production mais aussi dans le langage. Ainsi, à l'occasion du débrayage des ouvriers de

\footnotetext{
42 Robert Tremblay, «Un aspect de la consolidation du pouvoir d'État de la bourgeoisie coloniale: La législation anti-ouvrière dans le Bas-Canada, 1800-1850», Labour/Le Travailleur (Saint-Jean, T.-N.), 8/9 (automne/printemps 1981-1982), 247 ss.

${ }_{43}$ Ces inculpés étaient Jacques Bezeau, Jacques Lévesque, Olivier Lévesque, Jean-Baptiste Marcoux et Élie Simard, tous journaliers oeuvrant dans l'industrie de la construction navale.

44 ANQ-Q, Cour des sessions de la paix, district de Québec, 16 janvier 1841.

45 Archives de la ville de Québec, Série Conseil et procès-verbaux, 14 décembre 1840.

46 Canada, province du, Assemblée législative, Statuts, 1841, c. 26.

47 Robert Tremblay, «Un aspect de la consolidation du pouvoir d'État de la bourgeoisie coloniale...», 250.
} 
la construction navale, les marchands réunis au sein du Quebec Board of Trade se prononcèrent, au nom de la liberté de commerce, contre toute forme de cessation de travail, ce à quoi un charpentier de navire rétorqua:

\begin{abstract}
Permettez-moi au nom des charpentiers de cette ville [d'attester] de la justice de la démarche qu'ils ont adoptée de ne pas donner leur travail pour le mince salaire qu'on leur offre. Les charpentiers sont bien persuadés que la réduction que les maîtres de chantiers veulent introduire ne vient d'aucune diminution dans l'ouvrage ni dans le prix qu'ils auront; mais qu'ils veulent avoir pour rien le travail et les sueurs des ouvriers [...]. C'est un des constructeurs de vaisseaux les mieux fondés de Saint-Roch qui a été le premier à parler de réduire les gages à 3 shillings et qui a entraîné les autres à en faire autant; preuve que ce n'est pas la nécessité qui a amené cette réduction [des salaires]. ${ }^{48}$
\end{abstract}

Comme on le voit, la grève pousse à la réflexion et mène à l'élaboration de deux systèmes de pensée qui vont de plus en plus refléter l'antagonisme fondamental entre les forces du salariat et celles du capital.

Dans l'épreuve de force qui s'était établie en décembre 1840, la bourgeoisie de Québec avait cherché à s'adresser directement à la communauté ouvrière du faubourg Saint-Roch. Mais, comme son audience auprès des masses était faible, elle choisit de mettre à contribution les talents d'un intellectuel canadien-français, Étienne Parent. S'inspirant des économistes libéraux, tels Adam Smith et Jean-Baptiste Say, Parent élabora un discours qui avait pour base la théorie de l'équilibre naturel entre l'offre et la demande. Faisant de la libre concurrence entre fabricants le principe régulateur du prix des salaires, il déclarait en substance:

La seule [initiative] efficace et salutaire contre les exigences des chefs d'industrie est la concurrence entre eux, laquelle se règle sur la somme de travail dont ils ont besoin, combinée avec le nombre d'ouvriers sur place $[\ldots]$. Le bas prix de la main-d'oeuvre fera mettre beaucoup d'ouvrage sur les chantiers, et la grande somme d'ouvrage à faire, fera hausser les gages. ${ }^{49}$

Déplorant la coalition des ouvriers de la construction navale et l'associant à un geste contre nature, il ajoutait: «Vous ne pourrez jamais empêcher les maîtres de faire tous les profits que le marché leur permet de réaliser.» ${ }^{50} \ddot{\mathrm{A}}$ cette logique de l'accumulation, développée par les idéologues du capital, se superposait tout un langage ségrégationniste à l'endroit de la classe ouvrière. Ainsi, après avoir dénoncé «l'impré-

\footnotetext{
48 Le Canadien (Québec), 7 décembre 1840.

49 Ibid., 4 décembre 1840.

50 Étienne Parent, «Considérations sur le sort des classes ouvrières», in J.-C. Falardeau, Étienne Parent, 1802-1873 (Montréal, 1974), 296-330.
} 
voyance et la fénéantise [sic]» des charpentiers de navires, MM. Jones et Massue, conseillers municipaux de Québec, avaient déclaré: «Ce qui élève le marchand au-dessus de l'ouvrier et de l'artisan c'est parce qu'il marche dans une voie sûre et honnête. ${ }^{51}$ Le 4 décembre 1840 , dès le déclenchement de la grève, la Gazette de Québec commentait l'événement et qualifiait les travailleurs en ces termes: «Labour is like merchandise; the seller and the buyer must be free, and the price is fixed according to the wants of the parties.»

Néanmoins, les ouvriers de la construction navale firent valoir, en réponse à ces différents discours de pouvoir et de domination, que la précarité de leur existence matérielle les plaçait déjà dans une situation d'inégalité par rapport aux maîtres sur le marché du travail, ce qui anéantissait d'un coup la loi de la symétrie entre le prix des salaires et l'offre en main-d'oeuvre. L'un d'eux rappelait: «L'opulence n'a aucune idée de la somme de misère qui existe en ce moment dans nos faubourgs. ${ }^{52}$ Finalement, face au mépris, les ouvriers de Saint-Roch opposaient l'argument de la dignité humaine: «[Nous ne sommes] point des serfs, mais des hommes conçus pour jouir de l'air et ne travailler que pour de justes rétributions de salaire et de liberté.» ${ }^{53}$

S'il ressort de ce bref survol de la pensée prolétarienne des éléments comme le droit des ouvriers à la propriété de leur travail ou le droit à la dignité humaine, il reste toutefois à étayer le contenu de cette pensée et à analyser les multiples déterminations (économiques, politiques et idéologiques) qui structurent sa formation.

\section{Conclusion}

En dépit de son caractère de masse, la grève des ouvriers de la construction navale est avant tout un acte défensif par lequel la résistance ouvrière a voulu mettre un frein à l'arbitraire patronal et, ainsi, borner l'extorsion du «sur-travail». Cette attitude de repli s'explique dans la mesure où la perspective d'un ralentissement de l'activité productive dictait aux travailleurs des chantiers de repousser à plus tard les grandes revendications à l'endroit du capital, comme la journée de dix heures. L'attente d'un moment favorable pour déclencher une action offensive est typique de ce qu'il est convenu d'appeler la «conscience ouvrière des conjonctures» ${ }^{54}$. Néanmoins, la seule pratique de la grève ne pouvait qu'enrichir la faible expérience accumulée par la classe ouvrière dans ce domaine.

51 Le Fantasque (Québec), 8 février 1841.

52 Fernand Ouellet, Histoire économique et sociale du Québec, II: 503.

53 Le Fantasque (Québec), 8 février 1841.

54 Cette «conscience ouvrière des conjonctures» est d'abord empirique du fait qu'elle est alimentée par l'expérience quotidienne des travailleurs. Ainsi, en observant la diminution dans le stockage des matériaux ou les interruptions momentanées de l'activité productive, les ouvriers de la construction navale pouvaient facilement anticiper les périodes de crises et de licenciement dans cette industrie. 
Plus qu'un appendice au mouvement de grève, la naissance de la Société des charpentiers de vaisseaux doit être interprétée comme la mise en oeuvre des capacités dirigeantes de la classe ouvrière à des niveaux non étatiques. En ce sens, les premiers syndicats prefigurent, selon l'expression de Gramsci, un contre-pouvoir ouvrier dans la société. Mais ce contre-pouvoir sera étouffé pendant encore plusieurs années ${ }^{55}$, car n'oublions pas que la grève, par son effet de rayonnement social, secrète son double: la crainte de la grève qui pousse l'État à adopter des mesures de dissuasion à l'endroit de la classe ouvrière et qui exhorte le patronat à faire usage d'un discours visant à éduquer les masses dans un esprit contraire à leur propre praxis.

Quoi qu'il en soit, la grève des ouvriers de la construction navale, survenue à Québec en 1840, nous révèle le désir d'affranchissement d'une grappe d'hommes qui, pour reprendre une phrase du poète Paul Chamberland, étaient liés au souverain caprice du Capital.

\footnotetext{
55 Il faut au moins attendre jusqu'en 1872, année où l'on reconnaît officiellement les syndicats ouvriers, pour mesurer la véritable portée de ce contre-pouvoir.
} 